\title{
The MUSE experiment at PSI: Status and Plans
}

Wolfgang Lorenzon*i

Randall Laboratory of Physics, University of Michigan, Ann Arbor, Michigan 48109-1040, USA

E-mail: lorenzon@umich.edu

The MUSE experiment at the Paul Scherrer Institut (PSI) is part of a suite of experiments that aim to resolve the proton radius puzzle. MUSE is particularly interesting because it attempts to determine the proton radius through simultaneous measurements of muon-proton scattering and electron-proton scattering, in addition to measuring these reactions with both positive and negative leptons. This not only reduces systematic uncertainties but also provides sensitivity to two-photon exchange contributions which may be responsible for some of the discrepancies seen in earlier experiments. MUSE has almost completed its commissioning phase and is scheduled to start data collection this fall. We report on the current status and plans of the MUSE experiment.

PACS: MUSE; elastic scattering; muon beam

The 21st international workshop on neutrinos from accelerators (NuFact2019)

August 26 - August 31, 2019

Daegu, Korea

\footnotetext{
*Speaker.

${ }^{\dagger}$ representing the E1027 collaboration
} 


\section{The Proton Radius Puzzle}

Measurements of the proton charge radius started in the 1950's using electrons scattering off hydrogen targets at the Stanford accelerator. Since the cross section for electron proton scattering depends of the electric $\left(\mathrm{G}_{E}\right)$ and magnetic $\left(\mathrm{G}_{M}\right)$ form factors, a technique called Rosenbluth separation was used to separate the contribution of the two form factors and to extract the slope of the electric form factor $\left(\mathrm{G}_{E}\right)$ as $\mathrm{Q}^{2}$ approaches zero, which is proportional to the charge radius. More recently, atomic physicists have been using spectroscopy methods to very precisely measure atomic transitions in the hydrogen atom. Since the exact values of the hydrogen's energy levels depend on the size of the proton, they compared their measurements with Lamb shift calculations that include corrections for the finite size of the proton to extract an indirect but very precise value for the proton charge radius. Until about a decade ago, the proton charge radius from electron proton scattering measurements and from electronic hydrogen spectroscopy had converged to $r_{p}=0.8768 \pm 0.0069 \mathrm{fm}$ [1]. However in 2010, the CREMA collaboration at the Paul Scherrer Institut (PSI) reported results from a novel experiment using muonic-hydrogen spectroscopy [2] that determined the proton charge radius to be $r_{p}=0.84184 \pm 0.00067 \mathrm{fm}$. Confirmation of this surprising result came three years later [3]. This more than five-standard-deviation difference [4], shown in Fig. 1, has become known as the proton radius puzzle [5].

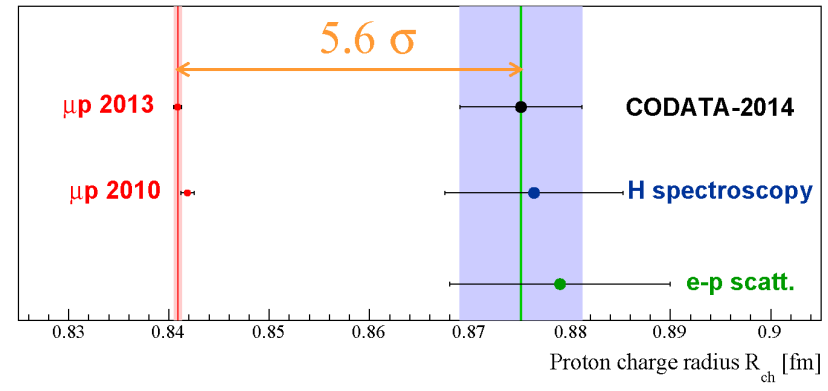

Figure 1: The proton radius measured with hydrogen spectroscopy and electron scattering is compared to that measured with muonic hydrogen. Radius from muonic hydrogen is $4.5 \%$ below the previous best value. That means it is $13 \%$ smaller (in volume) and therefore the proton is $13 \%$ denser than previously believed.

The roughly $4.5 \%$ smaller proton radius implied that the proton was about $13 \%$ denser than previously believed. This was not just an interesting result, but is directly related to the strength of the Strong Interaction (QCD), and therefore tests our theoretical understanding of the proton. Questions arose why exactly the muon and the electron give different proton radii, and it was speculated where the error, if there was in fact an error, could come from. Was it an experimental error in the $\mu p$ measurement, or in the $e p$ measurement? Both scenarios seemed unlikely. The $\mu p$ measurements had exquisite statistical and systematic precision, and for the ep measurements to be wrong, both the scattering and the hydrogen spectroscopy data had to be wrong. Or was it an error in the theoretical calculations? Although extracting a radius from hydrogen requires complex calculations, the calculations had been checked and re-checked without finding errors. So was it possible that indeed no mistakes had been made, and that the disagreement was due to new physics, possibly even due to physics beyond the Standard Model? Clearly, new data was needed to sort this out. 


\section{The MUSE experiment}

The MUon Scattering Experiment (MUSE) [6], located in the PiM1 area of the Paul Scherrer Institute (PSI) in Switzerland, is part of a suite of experiments that aim to resolve the proton radius puzzle. MUSE is particularly interesting because it attempts to determine the proton radius through muon proton scattering, which has only been done with low precision, simultaneously with electron proton scattering, in addition to performing these reactions with positive and negative leptons. This not only reduces systematic uncertainties but also provides sensitivity to two-photon exchange contributions which may be responsible for some of the discrepancies seen in earlier experiments. The electric form factor will be extracted from fits to the experimental cross section data. The experimental kinematics cover three beam momenta ${ }^{1}$ of about 115,153 , and $210 \mathrm{MeV} / \mathrm{c}$ and scattering angles in the range of $20^{\circ}-100^{\circ}$, corresponding to $Q^{2}$ of approximately $0.002(\mathrm{GeV} / \mathrm{c})^{2}-0.08(\mathrm{GeV} / \mathrm{c})^{2}$, the range of the form factor with greatest sensitivity to the radius [6].

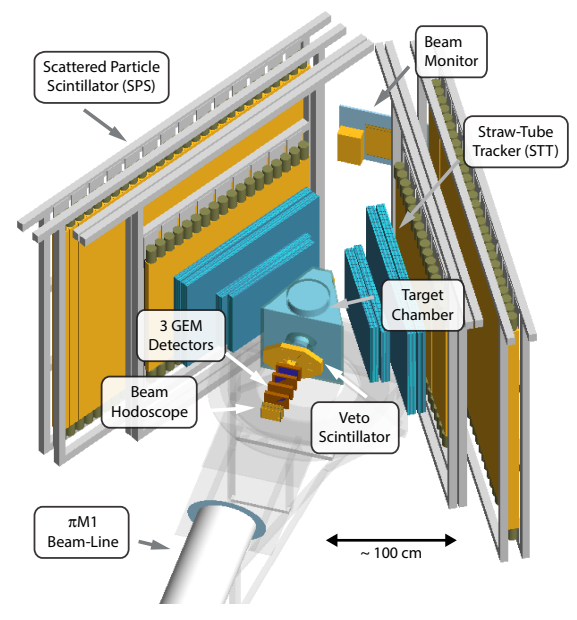

Figure 2: A schematic overview of the MUSE experimental setup is shown. The beam hodoscope determines the particle type through time-of-flight measurement. The GEM detectors measure the trajectories of the beam particles, and the veto scintillator tags beam halo particles. The beam monitor provides flux and timing information of the beam particles downstream of the target. The straw-tube trackers and the scattered particle scintillator planes provide track and timing information of the scattered particles.

MUSE is an unusual scattering experiment. While high precision electron scattering experiments typically use an intense primary beam, with scattered particles detected by a high-resolution, small solid-angle spectrometer, MUSE is using a low-intensity secondary beam that contains pions, electrons and muons where the scattered particles are detected by a large acceptance spectrometer. The resulting experimental setup is shown in Fig. 2.

The low beam intensity eliminates target density fluctuations from beam heating and allows identification of each individual beam particle; the electron contamination of the beam allows a simultaneous measurement of ep scattering for comparison with the muon scattering; and for a secondary beam it is easy to obtain essentially identical beams of both charge signs, which allows a precise determination of two-photon exchange (TPE) contributions.

\footnotetext{
${ }^{1}$ These particular beam momenta were chosen to allow clean separation of the pions, muons and electrons in the PiM1 beamline using RF timing information.
} 


\section{The $\mathrm{LH}_{2}$ Target}

At the heart of the experiment is a liquid hydrogen $\left(\mathrm{LH}_{2}\right)$ target that provides stable density and sufficient cooling power to minimize uncertainty in target length in order to allow cross section measurements at the sub-percent level [7]. Figure 3 demonstrates the performance of the $\mathrm{LH}_{2}$ target. The variation of the target temperature at the target cell operating temperature of $20.67 \mathrm{~K}$ during the first 72 -hr cooldown period was constant at the $0.01 \mathrm{~K}$ level. The $20.67 \mathrm{~K}$ target temperature corresponds to an operating pressure of about 1.1 bar and a target density of $0.070 \mathrm{~g} / \mathrm{cm}^{3}$ that was stable to within $0.02 \%$.

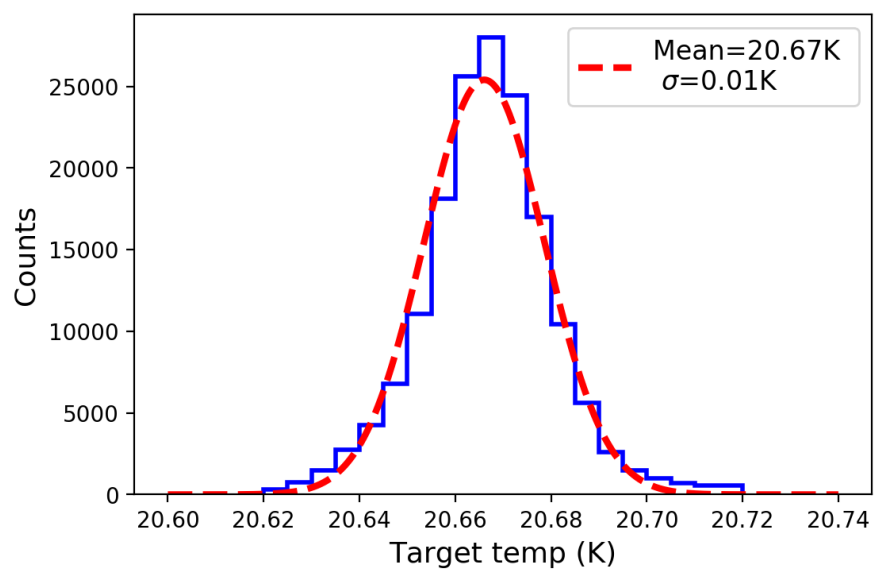

Figure 3: Histogram of target temperature values measured during the first cooldown period. The shape of the distribution is purely Gaussian with a mean of $20.67 \mathrm{~K}$ and a standard deviation of $0.01 \mathrm{~K}$.

\section{Current Status and Projections}

MUSE has completed 18 test running periods, including beam studies, detector development, and commissioning runs since 2012. The data collected during these periods agree well with detector simulations, and the apparatus has demonstrated reliable performance. Construction of the apparatus is almost complete. It is anticipated that commissioning of the detector hardware and experiment software will be completed during the November/December 2019 running period, so that first production data can be collected during that period. The remaining production data will be collected in 2020 and 2021. While a variety of physics results will be released in 2020, the final physics results are expected in 2022.

At that time, MUSE will have performed a high precision test of TPE for electrons and muons at low $\mathrm{Q}^{2}$, as well as a direct comparison of $e p$ to $\mu p$ elastic cross sections. The projected relative statistical uncertainty in the ratio of $\mu^{+} p$ to $\mu^{-} p$ elastic cross sections is expected to be below $3 \%$ with an overall systematic uncertainty of $0.2 \%$, while the projected relative statistical uncertainties in the ratio of the $e p$ to $\mu p$ elastic cross sections is below $2 \%$, with an overall systematic uncertainty of about $0.5 \%$. Note that the relative statistical uncertainties in the form factors are half as large as in the cross sections.

The absolute values of the radius extraction from electron and muon scattering will be of order $0.009 \mathrm{fm}$ each. However, the uncertainty in the difference of the radii extracted from $e p$ to $\mu p$ 
scattering will be of order $0.005 \mathrm{fm}$, since common systematic uncertainties cancel in the difference (see Fig. 4). The radius difference extraction is almost a factor two more sensitive than the absolute radius extraction, or almost factor ten better than the current discrepancy of about $0.034 \mathrm{fm}$.

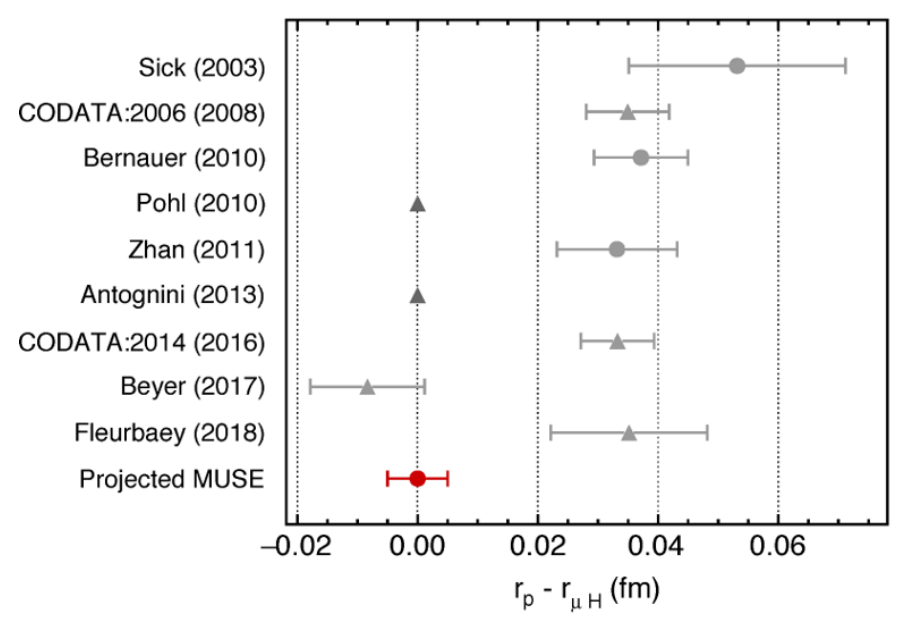

Figure 4: A summary of some recent proton charge radius determinations, relative to the muonic hydrogen result, along with the expected MUSE result, arbitrarily placed at 0 .

In summary, the discrepancy between muonic and electronic measurements remains a serious problem, although very recent electron scattering and spectroscopy results seem to gravitate towards the muonic hydrogen result. Several new results are expected in the coming years. Among those, MUSE will provide the first precise muon scattering result and will test the values for existing radius measurements. MUSE will also test the magnitude of the TPE contributions and provide a test of lepton universality by directly comparing the proton radii extracted from $e p$ to $\mu p$ scattering,

\section{References}

[1] P.J. Mohr, B.N. Taylor, and D.B. Newell, CODATA Recommended Values of the Fundamental Physical Constants: 2006, Rev. Mod. Phys. 80, (2008) 633.

[2] R. Pohl et al., The size of the proton, Nature 466, (2010) 213.

[3] A. Antognini et al., Proton Structure from the Measurement of 2S-2P Transition Frequencies of Muonic Hydrogen, Science 339, (2013) 417.

[4] P.J. Mohr, D.B. Newell, and B.N. Taylor, CODATA Recommended Values of the Fundamental Physical Constants: 2014, Rev. Mod. Phys. 88, (2016) 035009.

[5] R. Pohl, R. Gilman, G.A. Miller, and K. Pachucki, Muonic hydrogen and the proton radius puzzle, Annu. Rev. Nucl. Part. Sci. 63, (2013) 175.

[6] R. Gilman at al., Technical Design Report for the Paul Scherrer Institute Experiment R-12-01.1: Studying the Proton "Radius" Puzzle with $\mu p$ Elastic Scattering, arXiv:1709.09753v1 [physics.ins-det].

[7] P. Roy et al., A Liquid Hydrogen Target for the MUSE Experiment at PSI, Nucl. Instr. Meth. A949, (2020) 162874. 